\title{
LONG-TERM CRYOPRESERVATION CAN PREVENT REJECTION OF CANINE TRACHEAL ALLOGRAFTS WITH PRESERVATION OF GRAFT VIABILITY
}

\author{
H. Yokomise, MD \\ $\mathrm{K}$. Inui, MD \\ H. Wada, MD \\ M. Ueda, MD \\ S. Hitomi, MD
}

\begin{abstract}
We previously reported that cryopreservation of tracheas for 1 month was possible in a canine tracheal autotransplantation model with use of a preservative solution containing trehalose. Realizing that the allogenicity of many organs is decreased by freezing, we examined the possibility of immunosuppressant-free canine tracheal allotransplantation after long-term cryopreservation. Six to 10 rings of the trachea were removed from donor dogs $(n=12)$, immersed in the preservative solution, and cryopreserved in a deep freezer at $-85^{\circ} \mathrm{C}$ for $285 \pm 28$ days (cryopreservation group). Five rings of the mediastinal trachea of recipient dogs were removed. The cryopreserved tracheas were thawed and transplanted to replace the excised mediastinal tracheas. In a control group ( $n=6$ ), the graft was preserved in Euro-Collins solution at $10^{\circ} \mathrm{C}$ for 16 to 17 hours. Allotransplantation of tracheas was done as in the cryopreservation group. The anastomotic site and graft were covered with an omental pedicle in both groups. In the cryopreservation group, every animal, except one that was killed for pathologic examination, survived more than 2 months. All the grafts of this group were viable, and no stenosis or tracheomalacia was observed. In the control group, most of the animals died within 1 month of tracheal stenosis caused by rejection. These findings reveal that immunosuppressant-free canine tracheal allotransplantation was consistently possible after long-term cryopreservation of the graft in a preservative solution containing trehalose. This simple method could solve both donor shortage and immunosuppression problems. (J Thorac Cardiovasc Surg 1996; 111:930-4)
\end{abstract}

W e have reported that high-dose irradiation prevents rejection of canine tracheal allografts ${ }^{1}$ and that reliable 1-month cryopreservation of the trachea is possible in a trehalose cryopreservation (TC) solution. ${ }^{2}$ To solve two major problems of tracheal transplantation (rejection and preservation) simultaneously, we tried immunosuppressantfree tracheal allotransplantation after 1 month of cryopreservation in a preliminary experiment. However, most grafts were not viable, presumably because of severe damage derived from the combina-

From the Division of Thoracic Surgery, Chest Disease Research Institute, Kyoto University, Kyoto, Japan.

Received for publication May 15, 1995; accepted for publication August 9, 1995.

Address for reprints: H. Yokomise, MD, Division of Thoracic Surgery, Chest Disease Research Institute, Kyoto University, 53 Shogoin-Kawaharacho, Sakyo-ku, Kyoto, 606, Japan.

Copyright $(1996$ by Mosby-Year Book, Inc.

0022-5223/96 $\$ 5.00+0 \quad \mathbf{1 2 / 1 / 6 8 5 5 7}$ tion of irradiation and freezing (unpublished data), so we abandoned this approach.

With regard to cryopreservation itself, there have been many reports that the allogenicity of preserved allografts is decreased after cryopreservation. ${ }^{3-6}$ Furthermore, we have already reported that cryopreservation can preserve the viability of tracheal autografts (especially tracheal cartilage). ${ }^{2}$ Therefore, we tried immunosuppressant-free tracheal allotransplantation after long-term cryopreservation in $\mathrm{TC}$ solution.

\section{Material and methods}

Animals and anesthesia. Adult mongrel dogs were anesthetized with intramuscular ketamine $(10 \mathrm{mg} / \mathrm{kg})$ and intravenous sodium thiopental $(15 \mathrm{mg} / \mathrm{kg})$. After oral endotracheal intubation, the lungs were ventilated mechanically with a Harvard pump (Harvard Apparatus, Dover, Mass.) at a tidal volume of $20 \mathrm{ml} / \mathrm{kg}$ and a frequency of 20 breaths/min. Anesthesia was maintained with $50 \%$ oxygen, $50 \%$ nitrous oxide, and $1 \%$ halothane.

Groups and preservation of grafts. Six to 10 rings of trachea were removed and simply immersed in TC solu- 
tion containing Dulbecco's modified Eagle medium, 10\% dimethyl sulfoxide (DMSO), $20 \%$ fetal calf serum, and 0.1 $\mathrm{mol} / \mathrm{L}$ trehalose or Euro-Collins solution.

Group $A$. In the cryopreservation group (group A, $n=$ 12), the harvested tracheas were cryopreserved in TC solution and stored in a deep freezer at $-85^{\circ} \mathrm{C}$ for $285 \pm$ 28 days.

Group B. In the control group (group B, $n=6$ ), the harvested grafts were preserved in Euro-Collins solution at $10^{\circ} \mathrm{C}$ for 16 to 17 hours.

Allotransplantation of trachea. In group A, five rings of the mediastinal tracheas were resected and the cryopreserved tracheas were allotransplanted. Before transplantation the graft was thawed at $37^{\circ} \mathrm{C}$ in an incubator and rinsed with physiologic saline solution 10 times. In group B, the same procedure was done as in group A after the determined preservation time. Tracheal transplantation was done by the technique described previously. ${ }^{1}$ Briefly, five rings of mediastinal trachea were removed after the preparation of an omental pedicle graft, and a tracheal allograft was attached with 4-0 Prolene polypropylene (Ethicon Inc., Somerville, N. J.) continuous sutures. The anastomotic site and graft were covered with the omental pedicle graft. Intramuscular administration of cefazolin sodium (1 gm) was continued daily for 1 week after transplantation.

Follow-up bronchoscopic and histologic examinations. In both groups, the graft was examined by bronchoscopy 1 , 2,3 , and 4 weeks after operation. Thereafter, bronchoscopic examination was conducted every month. Before transplantation, one ring of the preserved trachea was resected and fixed in $10 \%$ formalin. When the experimental animals either died or were killed, transplanted grafts were removed and fixed in $10 \%$ formalin. Thus preserved grafts were examined histopathologically both before and after transplantation.

The experimental dogs were treated with humane care according to the guidelines outlined in "The Guide for the Care and Use of Laboratory Animals" prepared by the Institute of Laboratory Animal Resources and published by the National Institutes of Health (NIH Publication No. 86-23).

\section{Results}

Surviving animals (Table I). In the cryopreservation group, all animals $(n=12)$ survived for more than 2 months, except one animal killed for pathologic examination 7 days after the operation. In the control group, most animals died within 1 month mainly because of tracheal stenosis.

Macroscopic and gross findings. In the cryopreservation group, grafts were incorporated in all cases. Stenosis or tracheomalacia was not observed in any case (Fig. 1). In the control group, severe stenosis was observed in most cases (Fig. 2).

Histologic evaluation. After cryopreservation, the epithelium of the graft was depleted in all cases (Fig. 3, A). At 251 days after transplantation, the graft was covered with normal epithelium, and the
Table I. Results of current study

\begin{tabular}{crll}
\hline Groups (animal No.) & $\begin{array}{c}\text { Survival } \\
\text { (days) }\end{array}$ & Status of grafts & $\begin{array}{c}\text { Causes } \\
\text { of death }\end{array}$ \\
\hline Cryopreservation group & & & \\
cr-1 & 64 & Incorporated & Killed \\
cr-2 & 293 & Incorporated & Killed \\
cr-3 & 251 & Incorporated & Killed \\
cr-4 & 259 & Incorporated & Killed \\
cr-5 & 238 & Incorporated & Killed \\
cr-6 & 231 & Incorporated & Killed \\
cr-7 & 231 & Incorporated & Killed \\
cr-8 & 231 & Incorporated & Killed \\
cr-9 & 215 & Incorporated & Killed \\
cr-10 & 7 & Incorporated & Killed \\
cr-11 & 157 & Incorporated & Alive \\
cr-12 & 150 & Incorporated & Alive \\
Control group & & & \\
c-1 & 14 & Severe stenosis & Killed \\
c-2 & 37 & Severe stenosis & Killed \\
c-3 & 15 & Severe stenosis & Killed \\
c-4 & 14 & Severe stenosis & Killed \\
c-5 & 15 & Severe stenosis & Killed \\
c-6 & 7 & Edematous & Killed \\
\hline
\end{tabular}

viability of the tracheal cartilages was maintained (Fig. 3, B).

\section{Discussion}

Most candidates for tracheal transplantation have malignant diseases, and immunosuppressants cannot be given after the operation. To solve this basic problem, we developed a new procedure for tracheocarinal reconstruction in which we used the bifurcation of the right upper bronchus (as an autograft) to form a new carina. ${ }^{7}$ However, the results were not fully satisfactory and indications for this method will be limited. We next developed a high-dose irradiation method for tracheal transplantation. ${ }^{1}$ We found that 1000 Gy of gamma-ray irradiation prevented rejection of canine tracheal allografts. In addition, to solve the donor shortage problem, we developed a safe and reliable long-term preservation method for tracheas. ${ }^{2}$ With use of this method, we achieved consistent 1-month tracheal cryopreservation. Our next step was to try immunosuppressant-free tracheal allotransplantation after 1 month of cryopreservation as a preliminary experiment as mentioned in the former study. ${ }^{1}$ In this experiment, however, most grafts lost their viability presumably because of severe damage derived from the combination of irradiation and freezing (unpublished data) although graft viability had been pre- 

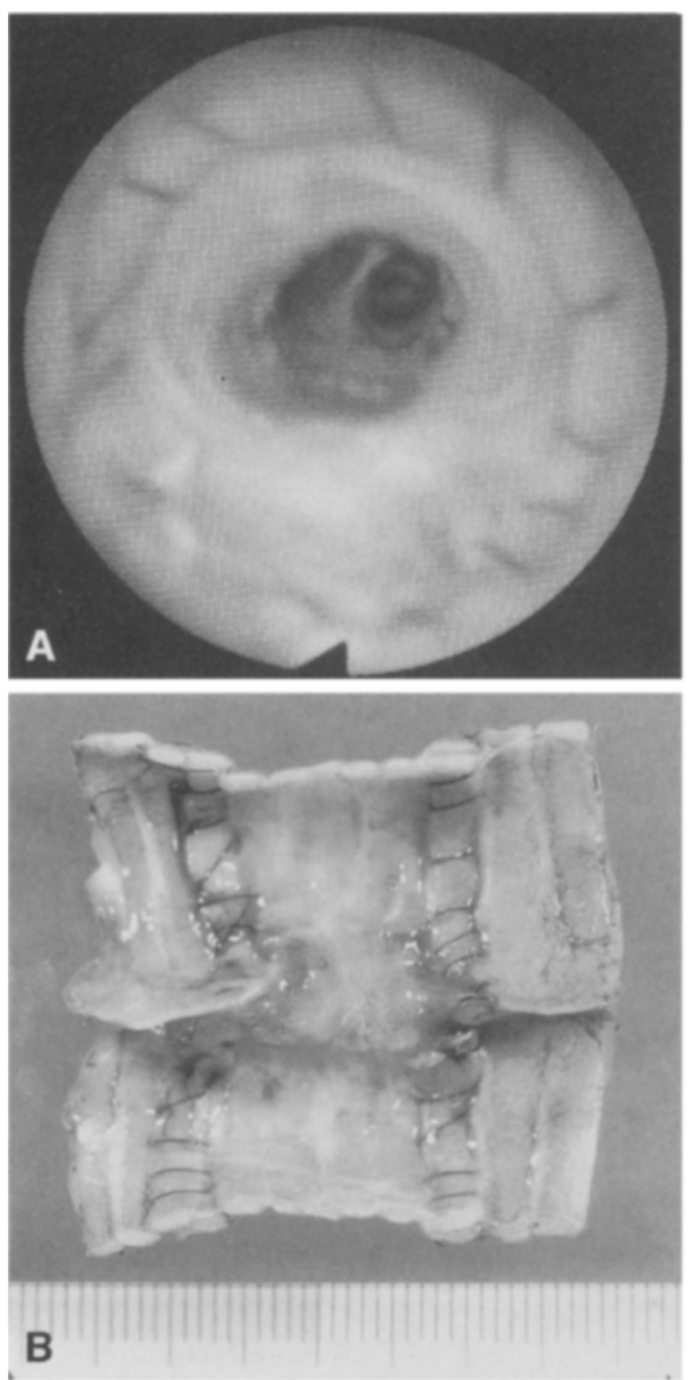

Fig. 1. Bronchoscopic (A) and macroscopic (B) findings 293 days after transplantation in cryopreservation group. There is no stenosis at anastomotic site or in graft. There is no evidence of atrophy in graft.

served in the previous studies. ${ }^{1,2}$ Therefore we gave up this approach.

There have been many reports that the allogenicity of preserved allografts can be decreased by freezing. ${ }^{3-5,6}$ Lupinetti and associates ${ }^{8}$ reported that cryopreservation denuded heart valves of endothelium and thus might decrease the allogenicity of the graft. We have already reported that the epithelium of the trachea plays an important role in graft rejection after transplantation. ${ }^{1}$ Thus we hypothesized that if after cryopreservation either the structure or the function of the tracheal epithelium is depleted, immunosuppressant-free tracheal allo-
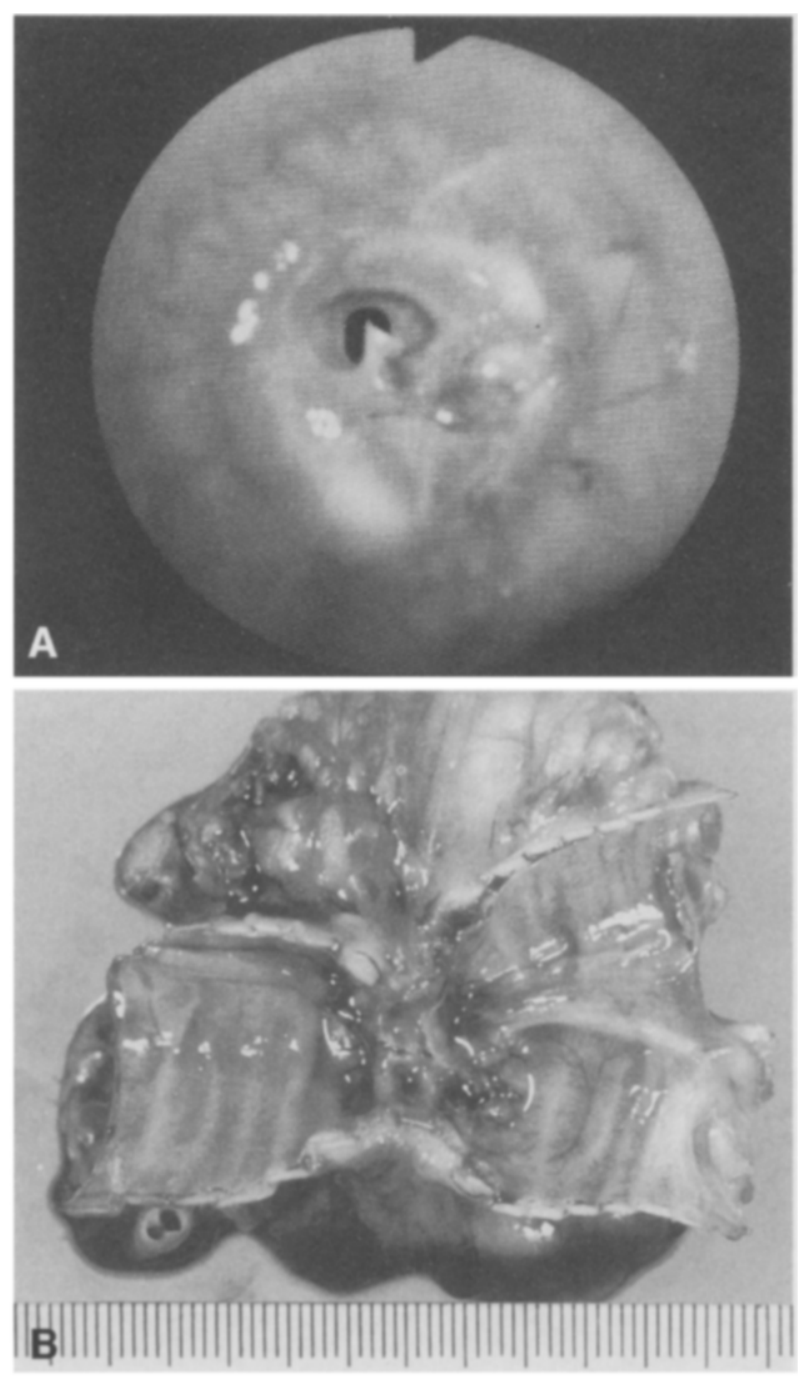

Fig. 2. Bronchoscopic (A) and macroscopic (B) findings 21 days after transplantation in control group. Marked stenosis was observed at anastomotic site and in graft.

transplantation after long-term cryopreservation might be possible, as it is after high-dose irradiation of the grafts. In fact, after 9 months of cryopreservation in TC solution, the epithelium of the trachea was depleted, and these grafts survived more than 2 months with preservation of graft viability. Probably the grafts that had lost epithelium after cryopreservation also lost allogenicity and became incorporated into the recipient's trachea. In this experiment, depletion of the epithelium must have played the most important role in the success of tracheal transplantation without immunosuppressants. It has been reported that the cartilage of the trachea has 

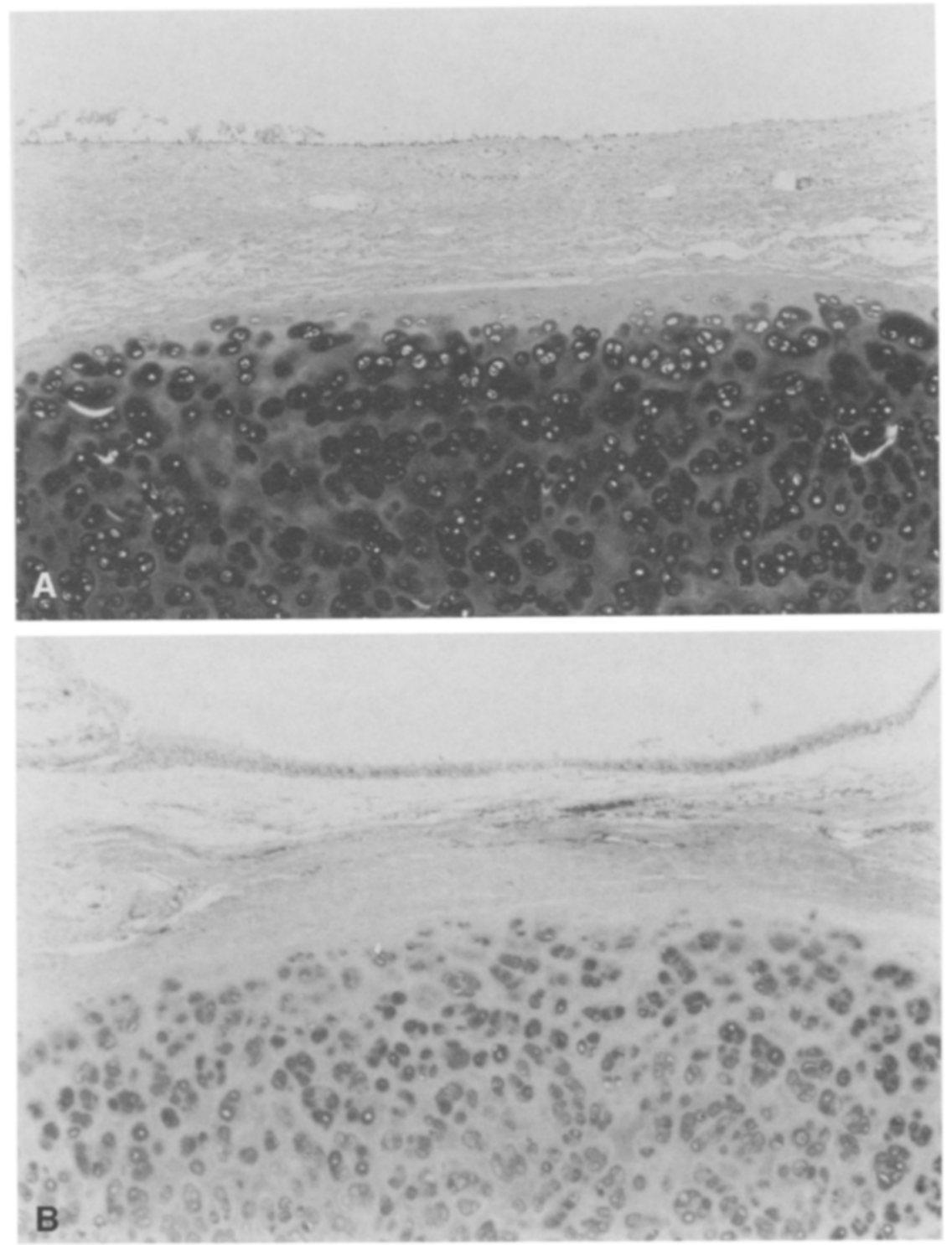

Fig. 3. A, Histologic findings of tracheal graft cryopreserved for 9 months. Epithelium has been depleted. However, viability of tracheal cartilage is maintained (original magnification $\times 40$ ). $\mathbf{B}$, Histologic finding of transplanted graft from animal killed 251 days after operation. Normal epithelium covers surface of graft, and viability of tracheal cartilages is maintained (original magnification $\times 40$ ).

low immunogenicity ${ }^{9}$ and can remain viable after cryopreservation. ${ }^{2,10}$ In this study, after cryopreservation of trachea in TC solution, depletion of the epithelium (modulation of allogenicity) and preservation of the cartilage (preservation of the architecture) were both achieved, and immunosuppressant-free allotransplantation of the trachea was successful.

Trehalose is a nonreducing disaccharide that protects the cell membrane from various stresses. ${ }^{11} \mathrm{We}$ recently developed new solutions for lung preserva- tion with satisfactory results. ${ }^{12,13}$ TC solution consists of Dulbecco's modified Eagle medium, 10\% DMSO, 20\% fetal calf serum, and $0.1 \mathrm{~mol} / \mathrm{L}$ trehalose. DMSO is a cryoprotectant that can penetrate cell membranes, whereas trehalose is a cryoprotectant that cannot penetrate cell membranes. The combined use of these different types of cryoprotectants has been reported to exhibit a synergistic effect in cryopreservation. ${ }^{14,15}$ Therefore we developed a solution that contained both DMSO and 
trehalose and found that 1-month cryopreservation of tracheas with this TC solution was consistently successful. ${ }^{2}$ The current study revealed that 9 -month cryopreservation is possible with the use of TC solution. Although the basic mechanism of the effect of TC solution has not been elucidated in our two experiments, TC solution enables immunosuppressant-free tracheal transplantation after long-term cryopreservation. The question of how long a tracheal graft must be cryopreserved for depletion of its allogenicity remains to be answered.

This approach has solved two basic problems of tracheal transplantation: modulation of allogenicity and reliable long-term preservation. With this simple method, we can stockpile tracheal grafts in regional transplant centers, replace grafts when needed, and offer cryopreserved tracheas as biomaterial when required. In summary, a new solution containing DMSO and trehalose can inhibit canine tracheal allogenicity and maintain the viability of the grafts after long-term cryopreservation.

\section{REFERENCES}

1. Yokomise $\mathrm{H}$, Inui $\mathrm{K}$, Wada $\mathrm{H}$, et al. High-dose irradiation prevents rejection of canine tracheal allografts. J Thorac Cardiovasc Surg 1994;107:1391-7.

2. Yokomise $H$, Inui $K$, Wada $H$, Hasegawa $S$, Ohno $N$, Hitomi S. Reliable cryopreservation of trachea for one month in a new trehalose solution. J Thorac Cardiovasc Surg 1995;110: 382-5.

3. Friedlaender GE. The antigenicity of preserved allografts. Transplant Proc 1976;8(suppl):195-200.

4. Hirase Y, Kojima T, Uchida M, Takeishi M. Cryopreserved allogeneic vessel and nerve grafts: hind-limb replantation model in the rat. J Reconstr Microsurg 1992;8:437-43.

5. Ingham E, Matthews JB, Kearney JN, Gowland G. The effects of variation of cryopreservation protocols on the immunogenicity of allogeneic skin grafts. Cryobiology 1993; 30:443-58.

6. Takeishi M, Hirase Y, Kojima T. Experimental study of cryopreserved allogeneic transfer of vessel: preliminary report. Microsurgery 1994;15:55-62.

7. Yokomise H, Inui K, Wada H, Hitomi S. A new method for carinal reconstruction: an experimental study. Thorac Cardiovasc Surg 1991;39:214-7.

8. Lupinetti FM, Tsai TT, Kneebone JM, Bove EL. Effect of cryopreservation on the presence of endothelial cells on human valve allografts. J Thorac Cardiovasc Surg 1993;106: 912-7.

9. Bujia J, Wilmes E, Hammer C, Kastenbauer E. Tracheal transplantation: demonstration of HLA class II subregion gene products on human trachea. Acta Otolaryngol (Stockh) 1990; 110:149-54.

10. Deschamps C, Trastek VF, Ferguson JL, et al. Cryopreservation of canine trachea: functional and histological changes. Ann Thorac Surg 1989;47:208-12.

11. Crowe JH, Crowe LM. Preservation of membranes in anhydrobiotic organisms: the role of trehalose. Science 1984;223: 701-3.

12. Hirata T, Fukuse T, Liu C, et al. Effects of trehalose in canine lung preservation. Surgery 1994;115:102-7.

13. Bando T, Kosaka S, Liu C, et al. Effects of newly developed solutions containing trehalose on twenty-hour canine lung preservation. J Thorac Cardiovasc Surg 1994;108: 92-8.

14. Honadel TE, Killian GJ. Cryopreservation of murine embryos with trehalose and glycerol. Cryobiology 1988;25: 331-7.

15. Terada T, Ashizawa K, Maeda T, Tsutsumi Y. Efficacy of trehalose in cryopreservation of chicken spermatozoa. Jpn J Anim Reprod 1989;35:20-5. 